\title{
Resultados del tratamiento a doce meses con ranibizumab para el edema macular diabético en la práctica clínica de rutina en México
}

\section{Twelve-month outcomes of ranibizumab for diabetic macular edema in routine clinical practice in Mexico}

\author{
Luis P. Orozco-Gómez'1, Leonor Hernández-Salazar', Vanesa Flores-Peredo², Alma L. Ortega-Villalobos ${ }^{3}$, \\ Sergio Aranda-Serna ${ }^{1}$, Luis Gerardo-Ferreyra ${ }^{1}$, Elisa Pérez-González ${ }^{1}$, Julio Pérez-Monjaraz ${ }^{1}$ y \\ Alejandra Sabina Ruiz-Franco ${ }^{4 *}$ \\ ${ }^{1}$ Centro Médico Nacional 20 de Noviembre; ${ }^{2}$ Hospital Lic. Adolfo López Mateos; ${ }^{3}$ Hospital $1{ }^{\circ}$ de Octubre; ${ }^{4}$ Hospital Regional Gral. Ignacio Zaragoza. \\ ISSSTE, Ciudad de México, México
}

\section{Resumen}

Objetivo: Evaluar los resultados de agudeza visual (AV) y grosor retiniano central (GRC) en pacientes con edema macular diabético (EMD) tratados con ranibizumab en la práctica clínica de rutina en México. Métodos: Se realizó un estudio prospectivo, abierto y de un solo grupo en 48 ojos de 34 pacientes con EMD con involucro central, en cuatro hospitales de alta especialidad del Instituto de Seguridad y Servicios Sociales de los Trabajadores del Estado (ISSTE). Los pacientes iniciaron con una dosis de carga de 3 inyecciones de ranibizumab $0.5 \mathrm{mg}$, seguido de monitoreo mensual con base en la AV y el GRC, para determinar la necesidad de retratamiento. La AV, el GRC, el puntaje en la Escala de severidad de la retinopatía diabética (DRSS) del ETDRS y el número de inyecciones se registraron al inicio y de manera mensual hasta los 12 meses. Resultados: La AV promedio, el GRC promedio y el puntaje promedio de la DRSS basales fueron $19( \pm 14)$ letras ETDRS, $362( \pm 98) \mu m$ y $50.59( \pm 7.9)$, respectivamente. El cambio promedio en AV a los 12 meses fue de $+8.6( \pm 4.3)$ letras $(p<0.001)$ respecto a la situación basal. El 39.58\% de los ojos $(n=19)$ ganó $\geq 10$ letras y el $37.50 \%(n=18)$ ganó $<10$ letras. El $4.16 \%$ $(n=2)$ se mantuvo estable y el 18.75\% ( $n=9)$ perdió < 10 letras. A los 12 meses, el cambio promedio en el GRC fue de $-85.34( \pm 32.1) \mu m(p<0.001)$. El puntaje promedio de la DRSS fue de $45.79( \pm 7.9)$ en el mes $12(p<0.001)$. Una media de 6.7 ( \pm 2.2$)$ inyecciones fue aplicada durante 12 meses. Conclusión: Los pacientes mostraron mejoría visual y anatómica después de recibir ranibizumab en la práctica clínica en México.

Palabras clave: Edema macular diabético. Ranibizumab. Anti-VEGF. Retinopatía diabética. Inyección intravítrea. México.

\section{Abstract}

Purpose: To evaluate visual acuity (VA) and central retinal thickness (CRT) outcomes in patients treated with ranibizumab for diabetic macular edema (DME) in a clinical setting in Mexico. Methods: A prospective, open-label, single arm study was performed on 48 eyes of 34 patients with center-involving DME at four high-specialty ISSSTE hospitals in Mexico. Patients

\section{Correspondencia:}

*Alejandra Sabina Ruiz-Franco

Calz. Gral. Ignacio Zaragoza, 1711

Col. Ejército Constitucionalista

Fecha de recepción: 04-05-2019

Fecha de aceptación: 20-06-2019

E-mail: asruiz222@gmail.com DOI: 10.24875/RMO.M19000085
Disponible en internet: 01-09-2019 Rev Mex Oftalmol. 2019;93(5):238-245

www.rmo.com.mx 0187-4519/@ 2019 Sociedad Mexicana de Oftalmología. Publicado por Permanyer México. Este es un artículo Open Access bajo la licencia CC BY-NC-ND (http://creativecommons.org/licenses/by-nc-nd/4.0/). 
initially received a loading dose of 3 injections of ranibizumab, followed by monthly monitoring based on VA and CRT to determine retreatment need. VA, CRT, ETDRS diabetic retinopathy severity scale (DRSS) score, and number of injections were recorded at baseline and monthly for 12 months. Results: The mean VA, mean CRT, mean DRSS score at baseline were 19 $( \pm 14)$ ETDRS letters, $362( \pm 98) \mu \mathrm{m}$ and $50.59( \pm 7.9)$. The mean VA change at 12 months was $+8.6( \pm 4.3)$ letters $(p<0.001)$ from baseline. The proportion of eyes that gained $\geq 10$ letters was $38.58 \%(n=19)$ and $37.50 \%(n=18)$ gained < 10 letters; $4.16 \%(n=2)$ had no change, and $18.75 \%(n=9)$ lost $<10$ letters. At month 12 , mean CRT change was $-85.34( \pm 32.1) \mu \mathrm{m}$ $(p<0,001)$ and the DRSS score was $45.79( \pm 7.9)(p<0.001)$. Patients received a mean of $6.7( \pm 2.2)$ injections over 12 months. Conclusion: Patients showed visual and anatomical improvement after receiving ranibizumab in a clinical setting in Mexico.

Key words: Diabetic macular edema. Ranibizumab. Anti-vascular endothelial growth factor. Diabetic retinopathy. Intravitreal injection. Mexico.

\section{Introducción}

La diabetes mellitus tipo 2 (DM2) afecta a aproximadamente 415 millones de personas en el mundo ${ }^{1}$, con una prevalencia estimada del $9.2 \%$ en la población adulta mexicana ${ }^{2}$. La retinopatía diabética (RD) es una complicación microvascular de esta enfermedad y está presente en el $70 \%$ de los pacientes con DM2 en Mé$\mathrm{xico}^{3}$. El edema macular diabético (EMD) ocurre en cualquier etapa de la RD y su incidencia aumenta con la duración de la $\mathrm{DM} 2^{4}$. Junto con la RD, el EMD constituye una de las principales causas de discapacidad visual y ceguera legal entre los adultos en edad productiva $^{4}$, lo que representa una carga para el sistema de salud mexicano en la actualidad.

La fotocoagulación con láser era el tratamiento estándar para el EMD antes de la aparición del tratamiento contra el factor de crecimiento endotelial vascular (VEGF), que mejora significativamente los resultados funcionales y anatómicos en comparación con el tratamiento con láser ${ }^{5}$. Varios estudios multicéntricos han evaluado la eficacia y la seguridad del ranibizumab intravítreo para el EMD. El estudio de fase II RESOLVE demostró la seguridad y la eficacia del ranibizumab mensual en el EMD durante 12 meses, lo que dio como resultado una ganancia de mejor agudeza visual corregida (MAVC) de +10.3 letras del ETDRS desde la situación basal ${ }^{6}$. El estudio de extensión de RESTORE demostró que el tratamiento individualizado con ranibizumab mejora y mantiene de manera efectiva los resultados funcionales y anatómicos durante más de 36 meses, con un número cada vez menor de inyecciones por año ${ }^{7}$. Además, el estudio RETAIN mostró la no inferioridad del régimen «tratar y extender» con ranibizumab versus ranibizumab por razón necesaria (PRN) durante 12 y 24 meses $^{8}$.

Sin embargo, los ensayos clínicos aleatorizados tienen criterios de inclusión y exclusión restrictivos, que podrían limitar su capacidad para representar poblaciones más amplias. Por lo tanto, los datos del mundo real son necesarios para evaluar la efectividad del tratamiento en el entorno clínico, así como para informar del impacto económico tanto de las enfermedades como de las intervenciones en países e instituciones sanitarias específicos ${ }^{9}$. En este sentido, varios estudios han confirmado la efectividad del ranibizumab en la práctica clínica habitual ${ }^{10-12} y$, hasta donde sabemos, no hay estudios que evalúen los resultados clínicos de ranibizumab en pacientes mexicanos con EMD.

\section{Propósito}

El objetivo de este estudio fue informar de los resultados visuales y anatómicos de ranibizumab en pacientes con EMD en un entorno del mundo real en México.

\section{Métodos \\ Diseño del estudio}

El presente estudio es un estudio multicéntrico intervencionista, prospectivo, de un solo grupo, abierto, realizado en cuatro hospitales de alta especialidad del Instituto de Seguridad y Servicios Sociales de los Trabajadores del Estado (ISSTE) en la Ciudad de México. Los pacientes recibieron inyecciones intravítreas de ranibizumab en uno o ambos ojos entre enero de 2015 y diciembre de 2015. El estudio fue aprobado por el Comité de Investigación del Centro Médico Nacional 20 de Noviembre del ISSSTE y se adhirió a los principios de la Declaración de Helsinki. Todos los participantes proporcionaron un consentimiento informado por escrito para la participación en el estudio. El estudio se registró con el número 507-2015. 


\section{Participantes}

Sujetos reclutados $\geq 18$ años de edad, hombres y mujeres, con alteración visual debida a EMD. Los pacientes tenían una MAVC basal entre 20/40 y 20/800 (equivalente Snellen), un grosor retiniano central (GRC) basal $\geq 250 \mu \mathrm{m}$ medido con Cirrus HD - tomografía de coherencia óptica (OCT), estudios fluorangiográficos retinianos y hemoglobina glucosilada $(\mathrm{HbA1c}) \leq 14 \%(130 \mathrm{mmol} / \mathrm{mol})$.

Los pacientes con presencia o sospecha de infección intraocular fueron excluidos del estudio, así como aquellos con isquemia miocárdica o eventos cerebrovasculares 6 meses antes de ingresar en el estudio. Los pacientes con tracción vitreomacular, isquemia macular, cirugía ocular dentro de los 3 meses previos al inicio del estudio, hemorragia vítrea y pacientes que requieren tratamiento concomitante con esteroides, también fueron excluidos.

Los pacientes se retiraron del estudio si requerían cirugía intraocular, se negaban a seguir participando o se perdieron durante el seguimiento.

\section{Intervención}

Los pacientes fueron tratados de acuerdo con las directrices institucionales del ISSSTE para el diagnóstico y tratamiento del $\mathrm{EMD}^{13}$. Se administró una dosis de carga de 3 inyecciones mensuales de ranibizumab $0.5 \mathrm{mg}$ (Lucentis ${ }^{\circledR}$, Novartis) a todos los pacientes, seguida de un monitoreo mensual que incluyó MAVC y OCT. El tratamiento se suspendía en caso de lograr una visión estable durante al menos 2 meses consecutivos y la resolución del edema. Los criterios de retratamiento incluyeron pérdida de $\geq 5$ letras del ETDRS, aumento del GRC $\geq 10 \%$ con respecto a la visita anterior y/o edema persistente en las imágenes de OCT, con inyecciones subsiguientes hasta que se lograra una MAVC de 20/20 y/o se mantuviera estable durante 2 meses y/o el GRC fuera de $<250 \mu \mathrm{m}$.

\section{Mediciones de resultados}

El criterio de valoración clínico primario fue el cambio medio de la MAVC (número de letras obtenidas en la cartilla de agudeza visual del ETDRS) desde la situación basal hasta los 12 meses. Los criterios de valoración secundarios incluyeron el cambio medio del GRC obtenido mediante Cirrus HD-OCT (Carl Zeiss Meditec) desde la situación basal hasta los 12 meses, el tiempo medio para lograr un GRC $<250 \mu \mathrm{m}$, el cambio en la puntuación media de la Escala de severidad de la retinopatía diabética (DRSS) del ETDRS, el número de inyecciones intravitreas recibidas durante 12 meses y los factores que afectan los resultados de MAVC y GRC.

\section{Análisis estadístico}

Realizamos cálculos de tamaño de muestra para obtener el tamaño de muestra deseado para el estudio. Se realizó un cálculo del tamaño de la muestra para obtener un contraste de hipótesis de la diferencia de medias para los grupos dependientes, una diferencia de medias de 6.2 letras y una desviación estándar de 6.1, según los resultados del estudio RETAIN en su grupo de tratamiento PRN, con una media basal de 63.4 letras \pm 11.15 , utilizando un valor alfa de 0.05 y un poder de 0.8 , lo que resultó en 41 ojos.

Se realizaron pruebas $t$ de Student para comparar las medias de las variables continuas y cuantitativas y la prueba de McNemar $\mathrm{X}^{2}$ para el análisis de las variables dicotómicas. Se usó una curva de supervivencia de Kaplan-Meier para medir la fracción de sujetos que mostraban un $\mathrm{GRC}<250 \mu \mathrm{m}$ durante 12 meses. Se realizaron análisis de regresión logística binaria para determinar la relación entre las variables independientes y los resultados categorizados para MAVC y GRC, es decir, ganancia de MAVC $>10$ y $<10$ letras, pérdida o no pérdida de letras y GRC $>2500<250$ $\mu \mathrm{m}$ en el mes 12. Se consideró un valor de $p=0.005$ como estadísticamente significativo para todos los análisis. Se utilizó el software SPSS versión $21\left(\right.$ IBM $\left.^{\circledR}\right)$.

\section{Resultados}

\section{Características basales}

Se incluyeron un total de 48 ojos de 34 pacientes. Diecinueve eran hombres (56\%), la edad media de todos los pacientes fue de 61.6 años, con una duración media de la DM2 de 16.8 años (rango 1-33) y un valor medio de $\mathrm{HbA} 1 \mathrm{c}$ de $7.9 \%$ (rango $5.5-13.1 \%$ ) (63 mmol/mol, 37-120). La media basal de MAVC fue de 19 letras (rango 5-47) y la media basal de GRC fue de $362 \mu \mathrm{m}$ (rango 215-839). El retiro del consentimiento fue la razón más común para el retiro del seguimiento; ocho pacientes fueron eliminados.

En la tabla 1 se resumen las características clínicas basales.

\section{Mejor agudeza visual corregida}

La media de MAVC después de 12 meses fue de 27.62 letras del ETDRS (rango 3-55). El cambio medio 


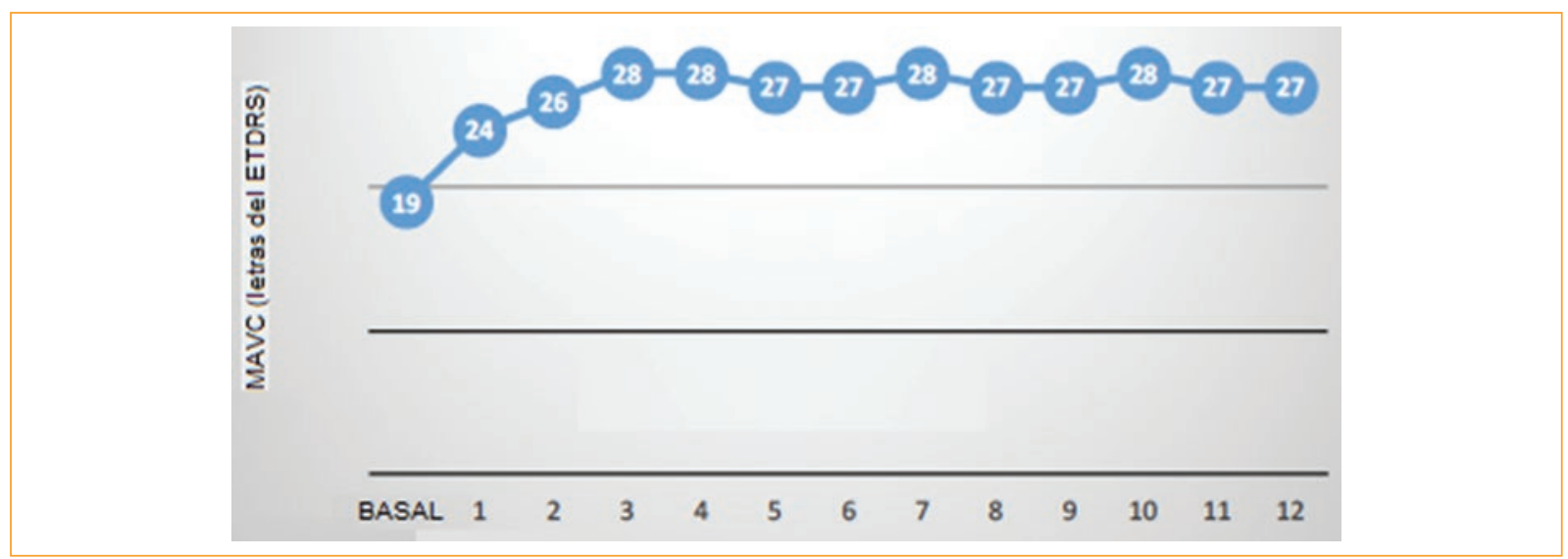

Figura 1. Cambio medio de agudeza visual desde la basal al mes 12.

MAVC: mejor agudeza visual corregida.

desde la situación basal hasta el mes 12 fue de +8.62 $( \pm 4.3)$ letras (Fig. 1), lo que muestra una diferencia estadísticamente significativa desde la situación basal $(p<0.001)$. El valor medio máximo de MAVC (28 letras) se observó después de la tercera inyección de ranibizumab, y se mantuvo hasta el mes 12. Los ojos se clasificaron en tres grupos según los resultados de MAVC en el mes 12 (Tabla 2). El $77.08 \%$ de los ojos experimentó mejoría visual $(n=37)$; el $39.58 \%$ del total $(n=19)$ ganó $\geq 10$ letras, mientras que $4.16 \%(n=2)$ no tuvo cambios y el $18.75 \%(n=9)$ perdió entre 1 y 3 letras.

\section{Grosor retiniano central}

En el mes 12, la media del GRC fue de $273.32 \mu \mathrm{m}$, lo que representa una reducción media significativa de $85.34( \pm 32.1) \mu \mathrm{m}(23.79 \%)$ desde la situación basal $(p<0.001)$. La figura 2 muestra la media del GRC obtenida cada mes desde la situación basal hasta el mes 12 .

La curva de supervivencia de Kaplan Meier (Fig. 3) demostró que el $41.3 \%$ de los ojos logró un GRC $<250$ $\mu \mathrm{m}$ después de la primera inyección intravítrea, el $62.5 \%$, después de la segunda dosis, y el $67 \%$, al tercer mes, con una mediana de 3.37 meses (IC 95\%: 2.42-4.38).

\section{Severidad de la retinopatía diabética}

La tabla 3 muestra la distribución de pacientes según la DRSS del ETDRS en la situación basal, a los 6 y 12 meses. En el mes 12 se observó una reducción numérica del porcentaje de pacientes con RD severa no proliferativa en comparación con la situación basal (de 20.83\% a $10.41 \%)$. De manera similar, el porcentaje de casos de RD proliferativa disminuyó de $18.74 \%$ a $8.33 \%$.
Tabla 1. Características basales

\begin{tabular}{|c|c|c|}
\hline Característica & Valor & Rango \\
\hline Edad, años $\pm D E$ & $61.65 \pm 7.9$ & $35-75$ \\
\hline \multicolumn{3}{|l|}{ Género, número } \\
\hline Hombres (\%) & $19(56 \%)$ & \\
\hline Mujeres (\%) & $15(44 \%)$ & \\
\hline Duración de la DM2, años & $16.8 \pm 5.4$ & $1-33$ \\
\hline MAVC, letras ETDRS & $19 \pm 14$ & $5-47$ \\
\hline $\mathrm{GRC}, \mu \mathrm{m}$ & $362 \pm 98$ & 215-839 \\
\hline $\mathrm{HbA1c}, \%$ (mmol/mol) & $7.9 \pm 2.2(63)$ & $5.5-13.1(37-120)$ \\
\hline $\begin{array}{l}\text { Ojos tratados } \\
\text { Ojo izquierdo }(\%) \\
\text { Ojo derecho }(\%) \\
\text { Total de ojos }\end{array}$ & $\begin{array}{c}27(56.2 \%) \\
21(43.8 \%) \\
48\end{array}$ & \\
\hline
\end{tabular}

DE: desviación estándar; GRC: grosor retiniano central; MAVC: mejor agudeza visual corregida.

Tabla 2. Clasificación de la mejor agudeza visual corregida (MAVC) final

\begin{tabular}{|l|c|c|}
\hline $\begin{array}{l}\text { Resultados de } \\
\text { MAVC }\end{array}$ & $\begin{array}{c}\text { Número de ojos } \\
\text { (rango de cambio de MAVC) }\end{array}$ & $\begin{array}{c}\text { Total de } \\
\text { ojos }(\%)\end{array}$ \\
\hline $\begin{array}{l}\text { Mejoría visual } \\
\geq 10 \text { letras ETDRS } \\
\leq 10 \text { letras ETDRS }\end{array}$ & $37(+4$ a +40$)$ & 77.08 \\
\hline MAVC estable & 19 & 39.58 \\
\hline Pérdida visual & 18 & 37.5 \\
\hline $\begin{array}{l}\text { +: ganancia de letras. } \\
- \text { pérdida de letras. }\end{array}$ & $9(-1$ a -3$)$ & 4.16 \\
\hline
\end{tabular}

La puntuación media de la DRSS del ETDRS mostró un cambio significativo de $50.59( \pm 7.9)$ a $45.79( \pm 7.9)$ en el mes $12(p<0.001)$. 


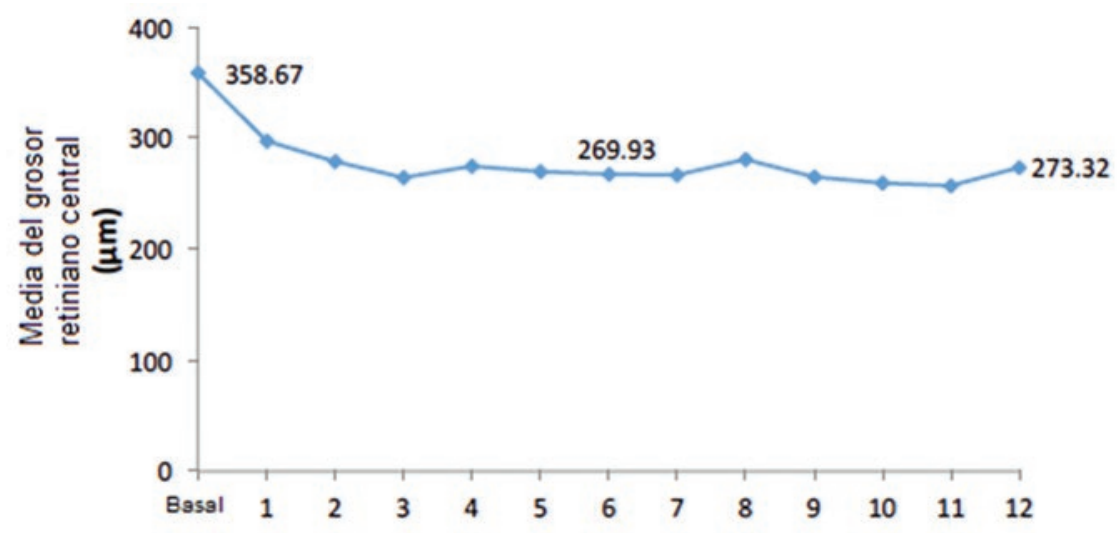

Figura 2. Cambio medio del grosor retiniano central desde el basal al mes 12 .

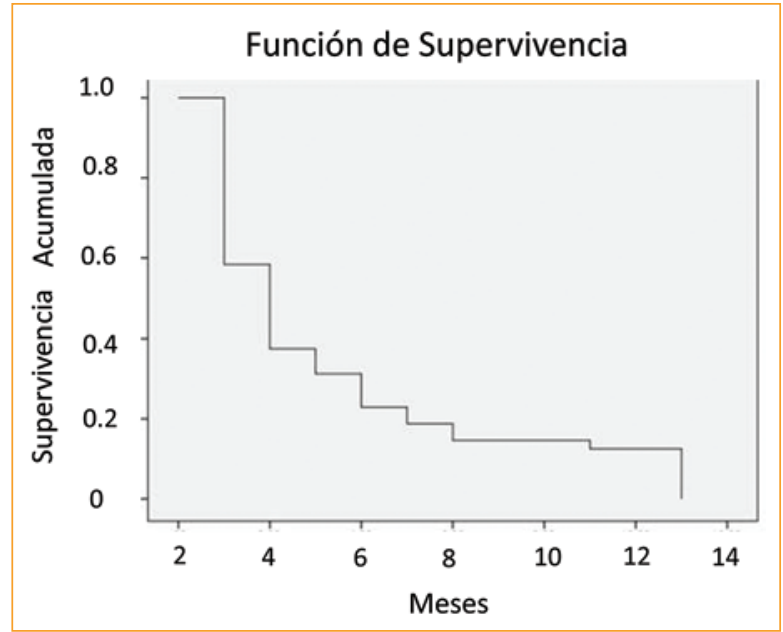

Figura 3. Curva de supervivencia de Kaplan Meier para la fracción de ojos con grosor retiniano central (GRC) $<250 \mu \mathrm{m}$. El $67 \%$ de los pacientes mostraron un GRC $<250 \mu \mathrm{m}$ en el mes 3, lo que destaca la importancia de administrar una dosis de carga para garantizar resultados positivos del tratamiento.

\section{Número de inyecciones}

Los pacientes recibieron una media de 6.7 inyecciones de ranibizumab por ojo tratado durante 12 meses. Este número incluye las 3 inyecciones mensuales de la fase de carga y las dosis posteriores de retratamiento PRN. El número total de inyecciones recibidas durante el estudio fue de 323 .

\section{Factores que afectan los resultados visuales y anatómicos}

El análisis de regresión logística binaria mostró que ninguna de las variables predictoras tuvo un impacto estadísticamente significativo en el resultado de MAVC a los 12 meses (Tabla 4).
Además, el análisis de regresión logística binaria no encontró una asociación significativa entre ninguna variable y la mejoría visual de $>10$ letras $0<10$ letras al final del estudio (Tabla 5).

Finalmente, el análisis de regresión logística binaria para el GRC final encontró que el GRC basal fue el único factor asociado significativamente con el grosor macular persistente en el mes $12(p=0.032,95 \% \mathrm{Cl})$ (Tabla 6).

\section{Discusión}

La alta prevalencia de DM2 es un problema de salud pública en México. EI EMD es una de las principales causas de pérdida visual en pacientes que viven con DM2 ${ }^{4}$. EI ISSSTE es una de las instituciones de salud más grandes de México, y brinda tratamiento con ranibizumab a pacientes con EMD clínicamente significativo. Este estudio es el primero en describir los resultados a los 12 meses de la terapia anti-VEGF en 48 ojos de 34 pacientes con EMD en México.

Las características basales en el presente estudio, incluida la duración de la DM2 y los niveles de HbA1c, fueron similares a las informadas en otros estudios clínicos. Nuestros resultados mostraron una ganancia media de 8.62 letras de MAVC desde la situación basal hasta el mes 12, que es similar a la informada en el estudio RETAIN (+7.4 letras del ETDRS en el mes 12 con ranibizumab y $0.5 \mathrm{mg} \mathrm{PRN})^{8}$. La mejoría visual máxima se observó después de la tercera inyección y se mantuvo en el mes 12. De manera similar, un estudio observacional que evaluó el uso de ranibizumab en la atención clínica de rutina en Suecia informó de ganancias visuales significativas en el $50 \%$ de los pacientes con EMD después de 4 meses de tratamiento, que se mantuvieron durante 12 meses $^{12}$. 
Tabla 3. Escala de severidad de retinopatía diabética del ETDRS en la situación basal, a los 6 y 12 meses

\begin{tabular}{|l|c|c|c|}
\hline Severidad de la retinopatía diabética & \multicolumn{3}{|c|}{ Número de pacientes (\%) } \\
\hline Nivel numérico del ETDRS & Basal & 6 meses & 12 meses \\
\hline Leve no proliferativa (35) & 0 & 0 & $5(10.41 \%)$ \\
\hline Moderada no proliferativa (47) & $24(50.00 \%)$ & $29(60.41 \%)$ & $33(68.75 \%)$ \\
\hline Severa no proliferativa (53) & $10(20.83 \%)$ & $12(25.00 \%)$ & $5(10.41 \%)$ \\
\hline Muy severa no proliferativa (57) & $5(10.41 \%)$ & $3(6.25 \%)$ & $1(2.08 \%)$ \\
\hline Proliferativa, sin alto riesgo (61) & $7(14.58 \%)$ & $3(6.25 \%)$ & $3(6.25 \%)$ \\
\hline Proliferativa de alto riesgo (71) & $2(4.16 \%)$ & $1(2.08 \%)$ & $1(2.08 \%)$ \\
\hline
\end{tabular}

Tabla 4. Análisis de regresión logística binaria del resultado de MAVC a los 12 meses

\begin{tabular}{|c|c|c|c|}
\hline Variable & Pérdida de letras del ETDRS & Sin pérdida de letras del ETDRS & p (IC 95\%) \\
\hline Género femenino, n (\%) & $6(31.6 \%)$ & $13(68.4 \%)$ & $0.29(1.3-2.34)$ \\
\hline Edad media, años \pm & $56.4 \pm 10$ & $62.5 \pm 6.7$ & $0.074(0.72-1.0)$ \\
\hline Duración media de DM2, años \pm & $18.0 \pm 7.29$ & $16.5 \pm 4.95$ & $0.915(0.82-1.2)$ \\
\hline Media de estadio basal de la RD, escala ETDRS \pm & 50.1 & 50.7 & $0.91(0.82-1.23)$ \\
\hline Media de creatinina, $\mathrm{mg} / \mathrm{dl} \pm$ & $1.62 \pm 0.78$ & $1.25 \pm 0.61$ & $0.087(0.73-9.7)$ \\
\hline Media basal deMAVC, letras del ETDRS \pm & $21 \pm 15.6$ & $18 \pm 13.7$ & $0.536(0.91-1.1)$ \\
\hline Media basal de $\mathrm{HbA} 1 \mathrm{c}, \% \pm(\mathrm{mmol} / \mathrm{mol})$ & $8.1 \pm 2.1(65)$ & $8.5 \pm 1.49(70)$ & $0.058(0.16-0.9)$ \\
\hline Media basal de GRC, $\mu \mathrm{m} \pm$ & $335 \pm 98$ & $364 \pm 99$ & $0.844(0.10-1.6)$ \\
\hline Número medio de nyecciones & 10 & 6 & $0.562(0.23-4.5)$ \\
\hline
\end{tabular}

\pm Desviación estándar.

DM2: diabetes mellitus tipo 2; GRC: grosor retiniano central; MAVC: mejor agudeza visual corregida; RD: retinopatía diabética.

Tabla 5. Análisis de regresión logística binaria de la mejoría de MAVC a los 12 mese

\begin{tabular}{|c|c|c|c|}
\hline \multirow{2}{*}{ Variable } & Media de MACV & Media de MACV & \multirow[b]{2}{*}{ p (IC 95\%) } \\
\hline & Cambio $>10$ letras & Cambio $<10$ letras & \\
\hline Edad media, años \pm & $62.41 \pm 7.0$ & $62.7 \pm 7.0$ & $0.626(0.92-1.1)$ \\
\hline Género masculino, $\mathrm{n}$ & 6 & 13 & $0.581(0.79-4.1)$ \\
\hline Duración media de la DM2, años \pm & $16.52 \pm 5.0$ & $16.55 \pm 5.0$ & $0.187(0.74-1.0)$ \\
\hline Media del estadio basal de la RD, escala ETDRS \pm & $50.91 \pm 6.6$ & $50.51 \pm 9.24$ & $0.980(0.90-1.1)$ \\
\hline Media basal de MAVC, letras del ETDRS \pm & $18.2 \pm 13.16$ & $19.75 \pm 14.54$ & $0.75(0.87-1.1)$ \\
\hline Media basal de $\mathrm{HbA} 1 \mathrm{c}, \% \pm(\mathrm{mmol} / \mathrm{mol})$ & $8.2 \pm 1.56(66)$ & $8.7 \pm 1.42(72)$ & $0.476(0.49-1.3)$ \\
\hline Media de creatinina, $\mathrm{mg} / \mathrm{dl} \pm$ & $1.0 \pm 0.48$ & $1.42 \pm 0.67$ & $0.057(0.42-0.98)$ \\
\hline Media basal de GRC, $\mu \mathrm{m} \pm$ & $340.52 \pm 61.81$ & $388.25 \pm 121.59$ & $0.102(0.97-1.39)$ \\
\hline Número medio de inyecciones & 10.47 & 6.0 & $0.102(0.97-1.39)$ \\
\hline Total de ojos & 19 & 18 & \\
\hline
\end{tabular}

\pm Desviación estándar.

DM2: diabetes mellitus tipo 2; GRC: grosor retiniano central; MAVC: mejor agudeza visual corregida; RD: retinopatía diabética. 
Tabla 6. Análisis de regresión logística binaria del resultado de GRC a los 12 meses

\begin{tabular}{|c|c|c|c|}
\hline Variable & $\mathrm{GRC}<250 \mu \mathrm{m}$ & $\mathrm{GRC}>250 \mu \mathrm{m}$ & p (IC 95\%) \\
\hline Edad media, años \pm & $60.37 \pm 8.07$ & $64.30 \pm 7.00$ & $0.691(0.88-32.4)$ \\
\hline Duración media de la DM2, años \pm & $17.5 \pm 5.1$ & $14.9 \pm 5.8$ & $0.115(0.96-1.34)$ \\
\hline Media de estadio basal de la RD, escala ETDRS \pm & $51.70 \pm 7.8$ & $47.61 \pm 7.8$ & $0.295(0.88-1.05)$ \\
\hline Media de creatinina, $\mathrm{mg} / \mathrm{dl} \pm$ & $1.38 \pm 0.10$ & $1.14 \pm 0.18$ & $0.848(0.86-1.18)$ \\
\hline Media basal de MAVC, letras del ETDRS \pm & $18.97 \pm 14.39$ & $19.07 \pm 13.26$ & $0.315(0.96-1.17)$ \\
\hline Media de $\mathrm{HbA} 1 \mathrm{c}, \% \pm(\mathrm{mmol} / \mathrm{mol})$ & $8.5 \pm 1.7(69)$ & $8.3 \pm 1.1(67)$ & $0.182(0.06-1.67)$ \\
\hline Media basal de GRC, $\mu \mathrm{m} \pm$ & $336.88 \pm 66.46$ & $420.38 \pm 136.4$ & $0.032(1.00-1.03)$ \\
\hline Total de ojos & 35 & 13 & \\
\hline
\end{tabular}

En cuanto a los resultados anatómicos, observamos una reducción media de $23.79 \%$ del GRC desde la situación basal hasta el mes 12 en el presente estudio. Esto es similar a la reducción del $27.09 \%$ que se muestra en el grupo de régimen PRN con ranibizumab del estudio RETAIN a pesar de que el GRC basal no es numéricamente similar en ambos estudios (362 $\mu \mathrm{m}$ frente a $432 \mu \mathrm{m}$, respectivamente) $)^{8}$. La curva de supervivencia de Kaplan-Meier encontró que después de recibir tres inyecciones mensuales, el $67 \%$ de los ojos tenía un GRC $<250 \mu \mathrm{m}$. Esto confirma la importancia de la dosis de carga para lograr el objetivo del tratamiento. Con un $41.3 \%$ de los pacientes que muestran una reducción favorable del GRC en el primer mes, podría ser razonable realizar una evaluación preliminar de la respuesta al tratamiento después de recibir la primera inyección de ranibizumab.

Se ha encontrado que los agentes anti-VEGF son beneficiosos en la RD proliferativa ${ }^{14}$. Además de la mejora visual y los resultados anatómicos en el EMD, en el presente estudio hubo numéricamente menos pacientes que presentaban RD severa no proliferativa y proliferativa a los 12 meses en comparación con la situación basal. La puntuación de la DRSS disminuyó significativamente, de 50.59 a $45.79(p<0.001)$. De manera similar, un análisis post-hoc de los estudios RISE y RIDE encontró que el $56.8 \%$ de los pacientes tratados con ranibizumab experimentaron una mejoría de 1 paso o más de la DRSS desde la situación basal hasta el mes 24 ; el $40 \%$ no tuvo cambios y el $3.2 \%$ experimentó un empeoramiento de la DRSS ${ }^{15}$. Además, el $17.1 \%$ de los pacientes en el grupo de $0.5 \mathrm{mg}$ de ranibizumab desarrollaron RD proliferativa durante 36 meses en comparación con el $39.1 \%$ de los pacientes en el grupo placebo, lo que sugirió que en los ojos tratados con ranibizumab, una mejoría mayor de la DRSS se relaciona con mejores resultados clínicos ${ }^{15}$.

En nuestro estudio, el tratamiento consistió en tres inyecciones mensuales seguidas de un régimen PRN según lo recomendado por las guías clínicas para el manejo del EMD en el ISSSTE ${ }^{13}$. El número medio de inyecciones por ojo tratado fue de 6.7 en 12 meses. Esto es consistente con el número de inyecciones informadas por el estudio RETAIN, en el que los pacientes del grupo PRN de ranibizumab recibieron una media de 7 inyecciones en 12 meses $^{8}$. En el estudio de extensión RESTORE, los pacientes recibieron un número decreciente de inyecciones por año, es decir, 7.9 el primer año; 3.9 el segundo, y 2.9 el tercero ${ }^{7}$. Por lo tanto, este régimen de tratamiento tiene el potencial de reducir la carga del tratamiento en las instituciones de salud.

Un gran estudio epidemiológico realizado en el Servicio de Oftalmología del Hospital General de México ( $n=13,670$ ) encontró que el $71 \%$ de los pacientes diabéticos tenía algún tipo de RD, de los cuales el $63 \%$ tenía la variante no proliferativa y el $37 \%$ RD proliferativa, con $16 \%$ de pacientes que presentan EMD en cualquier etapa de la $\mathrm{RD}^{3}$. En contraste, la prevalencia de RD en una gran cohorte de pacientes diabéticos ( $n=369,047$ ) en el Reino Unido fue del $43 \%$, de los cuales, el $11 \%$ tenía RD proliferativa ${ }^{16}$. Esto sugiere que la prevalencia de $\mathrm{RD}$ y $\mathrm{RD}$ proliferativa es mayor en la población mexicana, posiblemente debido a factores genéticos y de estilo de vida, así como a diferencias sustanciales en la detección y el manejo de la DM2 y la RD entre ambos países. La mayoría de los estudios clínicos aleatorizados en EMD se realizaron en países 
desarrollados; por lo tanto, es importante considerar si la variabilidad de la población ejerce un efecto sobre los resultados clínicos en el EMD. Aunque esta pregunta permanece fuera del alcance de este estudio, exploramos si las características basales de nuestra muestra de pacientes afectaron los resultados del tratamiento en el mes 12.

El análisis de regresión logística encontró una asociación significativa entre el GRC basal y el grosor macular persistente en el mes 12. Por lo tanto, podría ser razonable considerar el GRC basal para determinar la frecuencia óptima de seguimiento y retratamiento en cada paciente. Por otro lado, se ha encontrado que la MAVC basal es un factor predictivo para el resultado final de la MAVC ${ }^{17}$. En el presente estudio, sin embargo, no encontramos una asociación entre la agudeza visual inicial y final.

Las limitaciones del estudio incluyen el tamaño de la muestra. Se necesitan estudios adicionales con muestras más grandes para determinar los factores de predicción de los resultados de MAVC y GRC.

\section{Conclusión}

En este estudio, los pacientes experimentaron una mejoría visual y anatómica después de recibir inyecciones de ranibizumab en un entorno real. Observamos que el seguimiento mensual y el retratamiento individualizado, basados en criterios visuales y anatómicos, dan como resultado desenlaces clínicos potencialmente similares a los informados en los grandes estudios multicéntricos. La adherencia al régimen de PRN también podría ser útil para reducir la carga del tratamiento dentro de las instituciones de salud pública en México. Se necesitan más estudios para determinar la efectividad en una población más amplia, la mejoría de la calidad de vida relacionada con la visión y la rentabilidad de ranibizumab en las instituciones de salud mexicanas.

\section{Conflicto de intereses}

Los autores declaran que no existe conflicto de intereses en la realización y publicación de este estudio.

\section{Financiamiento}

Esta investigación no recibió ninguna subvención específica de agencias de financiamiento de los sectores público, comercial o sin fines de lucro.

\section{Responsabilidades éticas}

Protección de personas y animales. Los autores declaran que los procedimientos seguidos se conformaron a las normas éticas del comité de experimentación humana responsable y de acuerdo con la Asociación Médica Mundial y la Declaración de Helsinki.

Confidencialidad de los datos. Los autores declaran que han seguido los protocolos de su centro de trabajo sobre la publicación de datos de pacientes.

Derecho a la privacidad y consentimiento informado. Los autores han obtenido el consentimiento informado de los pacientes y/o sujetos referidos en el artículo. Este documento obra en poder del autor de correspondencia.

\section{Bibliografía}

1. International Diabetes Federation. IDF Diabetes Atlas. $7^{\text {th }}$ ed. Brussels, Belgium: IDF Executive Office; 2015. Disponible en: http://www.diabetesatlas.org. Accessed March 20, 2017.

2. Hernandez-Avila M, Gutierrez JP. Diabetes mellitus: la urgencia de reforzar la respuesta en políticas públicas para su prevención y control. Mexico: Instituto Nacional de Salud Pública; 2012.

3. Prado-Serrano A, Guido-Jimenez MA, Camas-Benitez JT. Prevalencia de retinopatía diabética en población mexicana. Rev Mex Oftalmol. 2009; 83(5):261-266

4. Klein R, Klein BE, Moss SE, Davis MD, DeMets DL. The Wisconsin epidemiologic study of diabetic retinopathy. IV. Diabetic macular edema. Ophthalmology. 1984;91(12):1464-74.

5. Boyer DS, Hopkins JJ, Sorof J, Ehrlich JS. Anti-vascular endothelia growth factor therapy for diabetic macular edema. Ther Adv Endocrinol Metab. 2013;4(6):151-69.

6. Massin P, Bandello F, Garweg JG, Hansen LL, Harding SP, Larsen M, et al. Safety and efficacy of ranibizumab in diabetic macular edema (RESOLVE Study): a 12-month, randomized, controlled, double-masked, multicenter phase II study. Diabetes Care. 2010;33(11):2399-405.

7. Mitchell P, Massin P, Bressler S, Coon CD, Petrillo J, Ferreira A, et al. Three-year patient-reported visual function outcomes in diabetic macular edema managed with ranibizumab: the RESTORE extension study. Curr Med Res Opin. 2015;31(11):1967-75.

8. Prünte C, Fajnkuchen F, Mahmood S, Ricci F, Hatz K, Studnička J, et al. Ranibizumab $0.5 \mathrm{mg}$ treat-and-extend regimen for diabetic macular oedema: the RETAIN study. Br J Ophthalmol. 2016;100(6):787-95.

9. Sherman RE, Anderson SA, Dal Pan GJ, Gray GW, Gerry W, Thomas Gross, et al. Real-world evidence - what is it and what can it tell us? N Engl J Med. 2016;375:2293-7.

10. Bertelmann T, Feltgen N, Scheffler M, Hufenbach U, Wiedon A, Wilhelm H, et al. Vision-related quality of life in patients receiving intravitreal ranibizumab injections in routine clinical practice: baseline data from the German OCEAN study. Health Qual Life Outcomes. 2016;14(1):132.

11. Ghanchi $F$, Hazel $C A$. South Asian diabetic macular oedema treated with ranibizumab (ADMOR)-real-life experience. Eye Lond Engl. 2016;30(1):133-8.

12. Granström T, Forsman $H$, Lindholm Olinder A, Gkretsis D, Eriksson JW, Granstam E, et al. Patient-reported outcomes and visual acuity after 12 months of anti-VEGF-treatment for sight-threatening diabetic macular edema in a real world setting. Diabetes Res Clin Pract. 2016;121:157-65.

13. Hernández-Salazar L, Aranda-Serna S, Orozco-Gómez LP, Azuara-Azuara C, Bracamontes-Barragán J, Collado-Solórzano A, et al. Guía para el diagnóstico y el tratamiento del edema macular diabético en derechohabientes del ISSSTE. Rev Esp Med Quir. 2015;20:321-7.

14. Osaadon P, Fagan XJ, Lifshitz T, Levy J. A review of anti-VEGF agents for proliferative diabetic retinopathy. Eye (Lond). 2014;28(5):510-20.

15. Ip MS, Zhang J, Ehrlich JS. The clinical importance of changes in diabetic retinopathy severity score. Ophthalmology. 2017;124(5):596-603.

16. Mathur R, Bhaskaran K, Edwards E, Lee H, Chaturvedi N, Smeeth L, et al. population trends in the Population trends in the 10-year incidence and prevalence of diabetic retinopathy in the UK: a cohort study in the Clinical Practice Research Datalink 2004-2014. BMJ Open. 2017;7(2):e014444.

17. Dugel PU, Hillenkamp J, Sivaprasad S, Vögeler J, Mousseau M-C, Wenzel A, et al. Baseline visual acuity strongly predicts visual acuity gain in patients with diabetic macular edema following anti-vascular endothelial growth factor treatment across trials. Clin Ophthalmol Auckl NZ. 2016;10:1103-10. 